\title{
Universality of Strength of Yukawa Couplings, Quark Singlets and Strength of CP Violation
}

\author{
G.C. Branco*, H.R. Colaço Ferreirał, A.G. Hesslerªnd J.I. Silva-Marcos ${ }^{\ddagger}$ \\ CFTP, Departamento de Física \\ Instituto Superior Técnico, Avenida Rovisco Pais, 1 \\ 1049-001 Lisboa, Portugal
}

March 25, 2022

\begin{abstract}
We analyse the strength of $\mathrm{CP}$ violation in an extension of the standard model with an extra $Q=-1 / 3$ vector-like singlet quark, in the framework of the hypothesis of universality of strength of Yukawa couplings connecting standard quarks. We show that the correct pattern of quark masses and mixing can be obtained, including the observed strength of CP violation.
\end{abstract}

PACS numbers : 12.10.Kt, 12.15.Ff, 14.65.Jk

*e-mail: gbranco@ist.utl.pt

$\dagger$ e-mail: hugoferreira@cftp.ist.utl.pt

‡e-mail: ahessler@cftp.ist.utl.pt

$\S$ e-mail: juca@cftp.ist.utl.pt 


\section{Introduction}

At present, there is no deep understanding of the observed pattern of fermion masses and mixing. In the framework of the Standard Model (SM), this pattern can be accommodated by adjusting the value of the Yukawa couplings, which is allowed, since the gauge symmetry does not constrain the flavour structure of the these couplings. There have many attempts at finding a rationale for the pattern of fermion masses and mixing [1].

An interesting suggestion for the flavour pattern of Yukawa couplings is the hypothesis of universal strength of Yukawa couplings (USY) [2], which assumes that the flavour dependence is all contained in their phases. The USY hypothesis, implemented within the SM, can accommodate most of the observed pattern of quark masses and mixing, with the notable exception of the strength of $\mathrm{CP}$ violation, measured by the rephasing invariant $I_{C P} \equiv\left|\operatorname{Im}\left(\mathrm{V}_{\mathrm{us}} \mathrm{V}_{\mathrm{cb}} \mathrm{V}_{\mathrm{cs}}^{*} \mathrm{~V}_{\mathrm{ub}}^{*}\right)\right|$. This is a remarkable achievement, since the fit of experimental data can be obtained even in the context USY ansätze 3] 4, where the USY phases are entirely fixed by the quark mass ratios, while the Cabibbo Kobayashi Maskawa mixing matrix (CKM) is predicted without free parameters. Prior to the measurement of the rephasing invariant angle $\gamma \equiv \operatorname{Arg}\left(-V_{11} V_{23} V_{13}^{*} V_{21}^{*}\right)^{C K M}$, of the standard unitarity triangle, the fact that USY within the SM cannot account for the observed strength of CP violation, was not a major drawback. One could always assume that New Physics (NP) contributed significantly to $\mathrm{CP}$ violation both in the Kaon and B-sectors, thus allowing for a small value of $I_{C P}$. This is no longer viable with the measurement of a large value of $\gamma[\underline{6}$, which does not receive significant contributions from NP [9]. However, NP can still give an important or even dominant contribution [7] to $\chi \equiv \operatorname{Arg}\left(-V_{23} V_{32} V_{22}^{*} V_{33}^{*}\right)^{C K M}$, which is small (of the order of a few percent) in the framework of the SM [7].

In this paper, we show that if one implements the USY hypothesis in the framework of an extension of the SM, where one vector-like [8] quark is introduced, one may fully account for the observed pattern of quark masses and mixing, including the size of $I_{C P}$. This paper is organized as follows. In the next section, we analyse a specific USY ansatz within the SM where the full CKM matrix is predicted as a function of the quark mass ratios, with no free parameters [5]. This analysis shows that the Ansatz is able to account for the pattern of quark masses and mixing, with the exception of the strength of CP violation. In section 3, we present an analytical study of a new USY ansatz in the framework of an extension of the SM, where one isosinglet vector-like quark is introduced. A numerical analysis of the same Ansatz is presented in section 4. This analysis indicates that, in the above extension of the SM, it is possible to account for the pattern of quark masses and mixing, including the strength of $\mathrm{CP}$ violation. Finally, we present our conclusions in section 5 .

\section{USY predictions within the SM}

In this section, we illustrate the USY predictions within the SM, in the framework of an Ansatz where the full CKM matrix is predicted in terms of quark mass ratios, with no free parameters [5]. Let us assume the following structure for the up and down quark mass matrices

$$
M_{u, d}=c_{u, d}\left(\begin{array}{ccc}
1 & 1 & e^{i(\alpha-\beta)} \\
1 & 1 & e^{i(\alpha)} \\
e^{i(\alpha-\beta)} & e^{i(\alpha)} & e^{i(\alpha)}
\end{array}\right)_{u, d}
$$

Since we have a total of three parameters for each sector, all quantities of the CKM matrix are exact functions of the quark mass ratios. To extract these, we proceed as follows. We define a squared mass matrix $H \equiv \frac{1}{9 c^{2}} M M^{\dagger}$ with trace normalized to 1 . Then, the two remaining invariants of $H$, are expressed in terms of the USY phases

$$
\begin{aligned}
& \operatorname{Det}[H] \equiv \delta=\frac{m_{1}^{2} m_{2}^{2} m_{3}^{2}}{\left(m_{1}^{2}+m_{2}^{2}+m_{3}^{2}\right)^{3}}=\frac{4^{2}}{9^{3}} \sin ^{4}\left(\frac{\beta}{2}\right) \\
& \chi[H] \equiv \chi=\frac{m_{1}^{2} m_{2}^{2}+m_{1} m_{3}+m_{2}^{2} m_{3}^{2}}{\left(m_{1}^{2}+m_{2}^{2}+m_{3}^{2}\right)^{2}}=\frac{4}{9^{2}}\left[\sin ^{2}\left(\frac{\alpha}{2}\right)+4 \sin ^{2}\left(\frac{\beta}{2}\right)+\sin ^{2}\left(\frac{\alpha-2 \beta}{2}\right)+2 \sin ^{2}\left(\frac{\alpha-\beta}{2}\right)\right]
\end{aligned}
$$


Taking into account the observed hierarchy of quark masses, Eq. (2) implies that both phases $\alpha, \beta$ have to be small. They can be expressed as explicit functions of the $\chi$ and $\delta$ :

$$
\begin{aligned}
& \sin ^{2}\left(\frac{\beta}{2}\right)=\frac{27}{4} \sqrt{\delta} \\
& \sin ^{2}\left(\frac{\alpha-\beta}{2}\right)=\frac{9^{2}}{4^{2}} \chi\left(\frac{1-2 \frac{\sqrt{\delta}}{\chi}}{1-\frac{27}{4} \sqrt{\delta}}\right)
\end{aligned}
$$

The Ansatz of Eq. (1) can be viewed as a specific perturbation of the so called "democratic ansatz", which is obtained in the limit $\alpha=\beta=0$. At a first stage of the perturbation, $\alpha \neq 0$, $\beta=0$ and the $u, d$ quarks are massless, so it corresponds to the chiral limit. In fact, it has been shown that the USY structures corresponding to the chiral limit can be classified into six exact solutions [4], so Eq. (1) with $\beta=0$ corresponds to one of these solutions [5]. At a second stage of the perturbation, $\beta \neq 0$ and the $u, d$ quarks acquire a mass. From Eqs. (2), 3), one obtains in leading order:

$$
|\alpha|=\frac{9}{2} \frac{m_{2}}{m_{3}} \quad ; \quad|\beta|=3 \sqrt{3} \frac{\sqrt{m_{1} m_{2}}}{m_{3}}
$$

It is also useful to introduce the parameters $\lambda$ and $A$ defined by

$$
\lambda=\sqrt{\frac{m_{1}}{m_{2}}} \quad ; \quad A=\frac{m_{2}^{2}}{m_{3} m_{1}}=O(1)
$$

It is clear that this choice of parameters was inspired by the Wolfenstein parametrization of the CKM matrix. Using Eq. (5), Eq. (4) translates into $|\alpha|=\frac{9}{2} A \lambda^{2},|\beta|=3 \sqrt{3} A \lambda^{3}$.

The quark mass matrices $H_{u, d} \equiv\left(\frac{1}{9 c^{2}} M M^{\dagger}\right)_{u, d}$ are diagonalized by:

$$
V_{u}^{\dagger} H_{u} V_{u}=D_{u}^{2} \quad ; \quad V_{d}^{\dagger} H_{d} V_{d}=D_{d}^{2}
$$

The unitary matrices $V_{u, d}$ can be written as:

$$
V_{u, d}=F \cdot W_{u, d} \quad ; \quad F=\left[\begin{array}{ccc}
\frac{1}{\sqrt{2}} & \frac{-1}{\sqrt{6}} & \frac{1}{\sqrt{3}} \\
\frac{-1}{\sqrt{2}} & \frac{-1}{\sqrt{6}} & \frac{1}{\sqrt{3}} \\
0 & \frac{2}{\sqrt{6}} & \frac{1}{\sqrt{3}}
\end{array}\right]
$$

The absolute values of the elements of the matrices $W_{u, d}$ are close to those of the identity and can be expressed in terms of the parameters $\lambda$ and $A$ as:

$$
\begin{aligned}
& \left|W_{12}\right|=\lambda(1+\ldots) \\
& \left|W_{23}\right|=\sqrt{2} A \lambda^{2}(1+\ldots) \\
& \left|W_{13}\right|=\frac{1}{\sqrt{2}} A \lambda^{3}(1+\ldots)
\end{aligned}
$$

Since we take the same flavour structure for the down and up quark sectors, the matrix $F$ cancels out in the computation of $V_{C K M}$ and one obtains:

$$
V_{C K M}=W_{u}^{\dagger} W_{d}
$$

This ansatz has the remarkable feature of giving $V_{C K M}$ in terms of the quark mass ratios, with no free parameters. In leading order one obtains:

$$
\begin{aligned}
& V_{12}^{C K M}=\sqrt{\frac{m_{d}}{m_{s}}}\left(1-\frac{3 i}{4} \frac{m_{s}}{m_{b}}-i \sqrt{3} \sqrt{\frac{m_{d}}{m_{s}}} \frac{m_{s}}{m_{b}}\right)-\sqrt{\frac{m_{u}}{m_{c}}} \\
& V_{23}^{C K M}=\sqrt{2} \frac{m_{s}}{m_{b}}\left(1+\frac{\sqrt{3}}{2} \sqrt{\frac{m_{d}}{m_{s}}}+\frac{3 i}{4} \frac{m_{s}}{m_{b}}+i \frac{\sqrt{3}}{2} \sqrt{\frac{m_{d}}{m_{s}}} \frac{m_{s}}{m_{b}}\right) \\
& V_{13}^{C K M}=\frac{-1}{\sqrt{2}} \frac{m_{s}}{m_{b}}\left(\sqrt{\frac{m_{d}}{m_{s}}}+2 \sqrt{\frac{m_{u}}{m_{c}}}\left(1+\frac{3 i}{4} \frac{m_{s}}{m_{b}}\right)-i \frac{\sqrt{3}}{2} \frac{m_{d}}{m_{s}} \frac{m_{s}}{m_{b}}\right) \\
& V_{22}^{C K M}=1-\frac{m_{d}}{2 m_{s}}-\frac{m_{u}}{2 m_{c}}+\sqrt{\frac{m_{d}}{m_{s}}} \sqrt{\frac{m_{u}}{m_{c}}}+\frac{3}{8}\left(\frac{m_{d}}{m_{s}}\right)^{2}-\left(\frac{m_{s}}{m_{b}}\right)^{2}
\end{aligned}
$$


where the signs in front of each of the mass ratio depend on the relative sign of the initial USY phase: 1 . These results for the USY ansatz are very encouraging as they lead to good phenomenological results for $V_{C K M}$ as a function of the quark mass ratios. One can see that in a first order approximation, the following sum rule holds in leading order:

$$
\left|\frac{V_{13}^{C K M}}{V_{23}^{C K M}}\right|=\frac{1}{2}\left|V_{12}^{C K M}\right|
$$

which is in agreement with experiment.

Having $V_{C K M}$ as a function of the quark mass ratios, one can also compute $I_{C P} \equiv \operatorname{Im}$ ( $\left.V_{12} V_{23} V_{22}^{*} V_{13}^{*}\right)^{C K M}$ as a function of the masses. The leading order term that one finds is

$$
\left|I_{C P}\right|=\frac{3 \sqrt{3}}{8}\left(\left|\left(\frac{m_{d}}{m_{s}}\right) \pm 2 \sqrt{3 \frac{m_{u}}{m_{c}}}\right|\right) \sqrt{\frac{m_{d}}{m_{s}}}\left(\frac{m_{s}}{m_{b}}\right)^{3}
$$

where again the sign depends on the relative sign of the initial USY phases. However, Eq. (12) illustrates the weak point of the USY hypothesis. From Eq. (12), one concludes that $\left|I_{C P}\right| \lesssim$ $o\left(\lambda^{9}\right) \approx 5 \times 10^{-7}$ which is too small to account for the experimental value $\left|I_{C P}\right| \cong 3 \times 10^{-5}$. The point is that this USY ansatz predicts correctly the values of $\left|V_{12}^{C K M}\right|,\left|V_{23}^{C K M}\right|,\left|V_{13}^{C K M}\right|$, $\left|V_{22}^{C K M}\right|$, but predicts a small value for $\gamma \equiv \operatorname{Arg}\left(-V_{11} V_{23} V_{13}^{*} V_{21}^{*}\right)^{C K M}$, which in turn leads to a small value for $I_{C P}$. The above statement may seem contradictory, since it is well known [10] that from the precise knowledge of four independent moduli of $V_{C K M}$, one can extract $\left|I_{C P}\right|$ using $3 \times 3$ unitarity. The contradiction is only apparent. The point is that a reliable extraction of $\left|I_{C P}\right|$ from $\left|V_{12}^{C K M}\right|,\left|V_{23}^{C K M}\right|,\left|V_{13}^{C K M}\right|,\left|V_{22}^{C K M}\right|$ (or from the moduli of the first two lines of $V_{C K M}$ ) requires a totally unfeasible precision in the knowledge of the above four moduli. A simple way of understanding this, is by realizing that the above extraction of $I_{C P}$ would be equivalent to deriving the area of the squashed unitarity triangle, corresponding to orthogonality of the first two lines of $V_{C K M}$, from the knowledge of its sides.

In general, it is not possible to generate a correct value of $I_{C P}$ within the USY framework implemented in the framework of the SM. Indeed, a numerical analysis reveals that, for all USY cases in the SM, not restricted to the particular form of Eq. (1), one is lead to $\left|I_{C P}\right|<10^{-6}$, which is too small compared to $\left|I_{C P}\right|_{\exp }$.

\section{USY with one extra vector-like quark}

\subsection{The Model}

The previous analysis provides motivation to implement the USY hypothesis in the framework of a minimal extension of the SM, consisting of the addition of the following fields to the SM spectrum:

- A vector-like down-type (i.e. $Q=-1 / 3$ ) quark $D$ added to the 3 generation left handed and right handed quark fields $Q_{L_{i}}, d_{R_{i}}, u_{R_{i}}$;

- A complex scalar Higgs singlet $S$, singlet under $S U(2) \times U(1)$.

In addition, to simplify the analysis, we impose a $Z_{2}$ symmetry under which all the fields of the SM transform trivially, while the new fields $D_{L}, D_{R}$ and $S$ are odd. With this symmetry, mass terms of the form $\overline{Q_{L_{i}}} \Phi D_{R}$ (i.e. the coupling to the SM Higgs) are absent. The Yukawa couplings and mass terms of $D$ are given by: $\bar{D}_{L}\left(f^{i} S+\widetilde{f}^{i} S^{*}\right) d_{R_{i}}+M \bar{D}_{L} D_{R}$. Upon spontaneous symmetry breaking, the Higgs bosons acquire VEV's: $\langle\Phi\rangle=v,\langle S\rangle=V_{S}$. It is clear that there are two scales in the model:

- the scale $v$ of electroweak $S U(2) \times U(1)$ breaking and

- the scale $V_{S}=\langle S\rangle$, which can be much larger $\left(\left|V_{S}\right| \gg v\right)$, since $\langle S\rangle$ does not break the $S U(2) \times U(1)$ gauge symmetry. The same applies to $M$, which can be much larger than $v$, since the mass term $\bar{D}_{L} D_{R}$ is a $S U(2) \times U(1)$ invariant.

\footnotetext{
${ }^{1}$ Althougth there are other terms of the same order in each of these $V_{C K M}$ elements, we only give here the terms which contribute to $I_{C P}$ in lowest order. Up to this order, one may take $V_{22}^{C K M}=1$. In addition, one may even vary the sign of each mass ratio $\pm \sqrt{\frac{m_{d}}{m_{s}}}, \pm \frac{m_{s}}{m_{b}}, \pm \sqrt{\frac{m_{u}}{m_{c}}}$, depending on the sign of the associated USY complex phase. However, despite this extra freedom, significant cancelations still occur in the leading order terms of $I_{C P}$, which remains small.
} 
We will assume a USY structure for the Yukawa couplings connecting standard quarks, so the up quark mass matrix has the form of Eq. (2), while the down quark mass matrix is given by:

$$
\mathcal{M}_{d}=c_{d}\left(\begin{array}{cccc}
1 & 1 & e^{i\left(\alpha_{d}-\beta_{d}\right)} & 0 \\
1 & 1 & e^{i \alpha_{d}} & 0 \\
e^{i\left(\alpha_{d}-\beta_{d}\right)} & e^{i \alpha_{d}} & e^{i \alpha_{d}} & 0 \\
p_{1} & p_{2} & p_{3} & \mu_{o}
\end{array}\right)
$$

where the $p_{i}$ are complex numbers given by $p_{i}=\frac{1}{c_{d}}\left(f^{i} V_{S}+\widetilde{f}^{i} V_{S}^{*}\right)$ and $\mu_{o}=\frac{1}{c_{d}} M$. In view of the above discussion, it is natural to assume that $O\left(\left|p_{i}\right|\right)=O\left(\mu_{o}\right) \gg 1$.

\subsection{The effective down quark mass matrix}

Next we evaluate how the presence of the vector-like quark $D$ affects the effective $3 \times 3$ down quark mass matrix connecting the standard quarks. Note that, it is this effective down quark mass matrix $H_{e f f}^{d}$ which, together with the up quark mass matrix, that generates the $3 \times 3$ CKM matrix connecting standard quarks.

In order to pursue our goal, we explicitly compute the effective $3 \times 3$ down quark mass matrix, resulting from Eq. (13) by integrating out the fourth heavy down quark2. We explain exactly how the extra vector-like quark modifies the pure USY hypothesis, and thus, gives a significant contribution to $\mathrm{CP}$ violation. We obtain in leading order:

$$
H_{e f f}^{d}=m m^{\dagger}-m \frac{P P^{\dagger}}{\mu^{2}} m^{\dagger}
$$

with

$$
\begin{aligned}
& m=\frac{1}{3}\left(\begin{array}{ccc}
1 & 1 & e^{i(\alpha-\beta)} \\
1 & 1 & e^{i(\alpha)} \\
e^{i(\alpha-\beta)} & e^{i(\alpha)} & e^{i(\alpha)}
\end{array}\right) \quad ; \quad P=\left(\begin{array}{c}
p_{1}^{*} \\
p_{2}^{*} \\
p_{3}^{*}
\end{array}\right) \\
& \mu^{2}=\left|p_{1}\right|^{2}+\left|p_{2}\right|^{2}+\left|p_{3}\right|^{2}+\mu_{o}^{2}
\end{aligned}
$$

where we have dropped the down-quark subscript $d$ and for convenience have normalized the trace of $m m^{\dagger}$ such that $\operatorname{Tr}\left(m m^{\dagger}\right)=1$. Note that the two terms of $H_{\text {eff }}^{d}$ in Eq. (14) are of the same order. This is a crucial point, since it makes possible to have a significant contribution to $\mathrm{CP}$ violation arising from the vector-like quark $D$. In particular, the contribution of the vector-like quark to $H_{e f f}^{d}$ does not decouple in the limit of large vector-like quark-mass, provided $V_{S}$ and $M$ in Eq. (13) are of the same order of magnitude. This is to be contrasted with the effect of the vectorlike quark on the tree-level flavour changing neutral currents (FCNC) which, although present in this class of models, are naturally suppressed by the ratio $\bar{m}^{2} / \bar{M}^{2}$, where $\bar{m}$ is the standard quark mass and $\bar{M}$ denotes the mass of the heavy vector-like quark. We shall return to this question in the sequel.

In addition (for our case), one can prove that $H_{\text {eff }}^{d}$ yields the same results as the full down $4 \times 4$ square mass matrix $\mathcal{M}_{d} \mathcal{M}_{d}^{\dagger}$ up to corrections of the order of $O\left(\left(\frac{m_{b}}{\bar{M}}\right)^{2}\right)$, with $\bar{M}$ denoting the mass of the extra vector-like down quark. We use $H_{\text {eff }}^{d}$ in order to have an analytical understanding of the effects of the vector-like quark. However, in our numerical analysis, we shall include the calculation with the full $4 \times 4$ square mass matrix $\mathcal{M}_{d} \mathcal{M}_{d}^{\dagger}$, its mass eigenvalues and diagonalization matrix.

\subsubsection{FCNC}

An important effect of the presence of extra vector-like quarks is the occurrence at tree level of flavour changing neutral currents (FCNC) and their associated electroweak precision measurements

\footnotetext{
${ }^{2}$ For convinience and instead of the dimensionfull effective matrix $\mathbf{H}_{e f f}^{d}$, we shall work with a dimensionless matrix $H_{e f f}^{d}$, defined as $H_{\text {eff }}^{d} \equiv \frac{1}{9 c_{d}^{2}} \mathbf{H}_{e f f}^{d}$.
} 
(EWPM), which place severe restrictions on this class of models. For our case, with one extra vector-like down quark, FCNC only occur in the coupling of the $Z$ with the down quarks:

$$
-\frac{g}{2 \cos \left(\theta_{W}\right)} \bar{d}_{L}^{\alpha} \gamma^{\mu} \mathcal{U}_{\alpha \beta} d_{L}^{\beta} Z_{\mu}
$$

where $\mathcal{U}$ is a $4 \times 4$ matrix computed from $\mathcal{V}$ the full $3 \times 4$ CKM matrix 3$]^{3} \mathcal{U}=\mathcal{V}^{\dagger} \mathcal{V}$. The CKM matrix $\mathcal{V}$ is obtained from $\mathcal{V}=V_{u}^{\dagger} \mathcal{K}_{d}$, where $V_{u}$ is the unitary matrix which diagonalizes the $3 \times 3$ (squared) up quark mass matrix $H_{u}$, and $\mathcal{K}_{d}$ is the $3 \times 4$ part of the unitary matrix $\mathcal{W}_{d}=\left(\begin{array}{c}\mathcal{K}_{d} \\ \beta_{d}\end{array}\right)$ which diagonalizes the full $4 \times 4$ (squared) down quark mass matrix $\mathcal{M}_{d} \mathcal{M}_{d}^{\dagger}$ in Eq. (13). Since $V_{u}$ is unitary, one has $\mathcal{V}=\mathcal{K}_{d}^{\dagger} \mathcal{K}_{d}$. The CKM matrix is not unitary, but its effective deviation from unitarity is very small, being naturally suppressed by the ratio $\bar{m}^{2} / \bar{M}^{2}$, where $\bar{m}$ is the standard quark mass and $\bar{M}$ denotes the mass of the heavy vector-like quark.

The most stringent EWPM is the branching ratio of $K^{+} \rightarrow \pi^{+} \nu \bar{\nu}$, which places a severe limit on the FCNC coupling $\mathcal{U}_{s d}$. This branching ratio, and other quantities sensitive to FCNC, which depend on the $\mathcal{U}_{i j}$, have been computed for models with vector-like down quarks [12]. In our case, we typically obtain values $\left|\mathcal{U}_{s d}\right|,\left|\mathcal{U}_{d s}\right| \leq \times 10^{-9},\left|\mathcal{U}_{b d}\right|,\left|\mathcal{U}_{d b}\right| \leq \times 10^{-8},\left|\mathcal{U}_{b s}\right|,\left|\mathcal{U}_{s b}\right| \leq \times 10^{-6}$. For these values, we find no significant effect on the associated EWPM.

\subsection{Extra CP violation from the vector-like quark}

In order to explicitly show the effect of the extra vector-like quark, we choose as an example: $\left(p_{1}, p_{2}, p_{3}, \mu\right)=(i k, i k,-k, k)$. For this case, we see that the dependence on $k$ cancels out and the extra term $\frac{P P^{\dagger}}{\mu^{2}}$ in Eq. (14) reduces to a simple expression:

$$
\frac{P P^{\dagger}}{\mu^{2}}=\frac{1}{4} d_{i} \Delta d_{i}^{*} \quad ; \quad d_{i}=\operatorname{diag}(i, i, 1) \quad ; \quad \Delta=\left(\begin{array}{ccc}
1 & 1 & 1 \\
1 & 1 & 1 \\
1 & 1 & 1
\end{array}\right)
$$

and therefore,

$$
H_{\text {eff }}^{d}=m m^{\dagger}-\frac{1}{4} m d_{i} \Delta d_{i}^{*} m^{\dagger}
$$

The diagonalization of this effective matrix is done in two steps. First, we use the result obtained in Eqs. (5, 7, 8), where we have expressed the diagonalization matrix $V=F \cdot W$ of $m m^{\dagger}$ as the product of a fixed unitary matrix $F$ and a unitary matrix $W$, whose absolute value is close to the identity and which can be parametrized à la Wolfenstein. The matrix $m$ is symmetric, thus we have $V^{\dagger} m V^{*}=d$, where $d$ is diagonal and real. All parameters, including the USY phases, the eigenvalues of $m m^{\dagger}$ and $W$, are given as expansions in a small parameter $\bar{\lambda}$, and as we chose $\operatorname{Tr}\left(m m^{\dagger}\right)=\operatorname{Tr}\left(d^{2}\right)=1$, we have for the leading order terms: $d=\operatorname{diag}\left(\overline{A \lambda}^{4}, \overline{A \lambda}^{2}, 1\right)$. Therefore, partially diagonalizing $H_{\text {eff }}^{d}$ we obtain

$$
H_{e f f}^{d} \longrightarrow \widetilde{H}_{e f f}^{d}=V^{\dagger} H_{e f f}^{d} V=d^{2}-\frac{1}{4} d W^{T} F^{T} d_{i} \Delta d_{i}^{*} F W^{*} d
$$

Note that we chose different symbols $\bar{\lambda}, \bar{A}$ instead of the original $\lambda, A$. This is necessary because the eigenvalues of $m m^{\dagger}$ do not coincide with those of $H_{e f f}^{d}$. Only the eigenvalues of $H_{\text {eff }}^{d}$ relate to the real physical masses. For simplicity, let us for now assume that $W=\mathbb{I}$, then in leading order we have

$$
\begin{aligned}
& \widetilde{H}_{\text {eff }}^{d}=d^{2}-\frac{1}{4} d\left(\begin{array}{rrr}
0 & 0 & 0 \\
0 & \frac{4}{3} & \frac{-\sqrt{2}(1+3 i)}{3} \\
0 & \frac{-\sqrt{2}(1-3 i)}{3} & \frac{5}{3}
\end{array}\right) d= \\
& =\left(\begin{array}{rrr}
\bar{A}^{2} \bar{\lambda}^{8} & 0 & 0 \\
0 & \frac{2}{3} \bar{A}^{2} \bar{\lambda}^{4} & \frac{(1+3 i)}{6 \sqrt{2}} \overline{A \lambda}^{2} \\
0 & \frac{(1-3 i)}{6 \sqrt{2}} \overline{A \lambda}^{2} & \frac{7}{12}
\end{array}\right)
\end{aligned}
$$

\footnotetext{
${ }^{3}$ In the case of the SM, the CKM matrix $\mathcal{V}$ coincides with an ordinary $3 \times 3$ unitary matrix $V$, and then, $\mathcal{U}$ is the $3 \times 3$ identity matrix, so there are no FCNC's at tree level.
} 
We see that to complete the total diagonalization of $H_{e f f}^{d}$, we have to diagonalize an extra $2 \times$ 2 Hermitian matrix. This will give an important contribution to $V_{C K M}$, since the extra term corresponds to two modifications:

- first, further diagonalization implies the transformation of the matrix $\widetilde{H}_{\text {eff }}^{d}$ in Eq. (20) with an additional diagonal unitary matrix $K=\operatorname{diag}\left(1,1, \frac{(1-3 i)}{\sqrt{10}}\right)$ and with an additional orthogonal matrix $O_{23}$ such that, in leading order

$$
D_{e f f}^{2}=O_{23}^{T} K^{*} \widetilde{H}_{e f f}^{d} K O_{23}=\left(\begin{array}{rrr}
\bar{A}^{2} \bar{\lambda}^{8} & 0 & 0 \\
0 & \frac{3}{7} \bar{A}^{2} \bar{\lambda}^{4} & 0 \\
0 & 0 & \frac{7}{12}
\end{array}\right)
$$

where $\mathrm{O}_{23}$ is given in leading order by

$$
O_{23}=\left(\begin{array}{lll}
1 & 0 & 0 \\
0 & 1 & \frac{2 \sqrt{5}}{7} \overline{A \lambda}^{2} \\
0 & -\frac{2 \sqrt{5}}{7} \overline{A \lambda}^{2} & 1
\end{array}\right)
$$

- and second, since the trace in Eq. (21) of the total effective matrix is no longer normalized to 1 , this implies that the parameters $\bar{\lambda}$ and $\bar{A}$ are slightly different from the original $\lambda, A$. We stress again, that this results from the fact that the eigenvalues of $m m^{\dagger}$ are not the same as the eigenvalues of $H_{e f f}^{d}$. From Eq. (21), it easy to conclude that, for this effective mass matrix, we now have in leading order

$$
\bar{\lambda}=\left(\frac{3}{7}\right)^{\frac{1}{4}} \sqrt{\frac{m_{1}}{m_{2}}} \quad ; \quad \bar{A}=\frac{7 \sqrt{7}}{6 \sqrt{3}} \frac{m_{2}^{2}}{m_{3} m_{1}}
$$

which will give a significant contribution to $V_{C K M}$. The masses, of course, refer to the down sector. The angle of the extra diagonalization matrix $O_{23}$ is, in leading order, equal to $\frac{\sqrt{5}}{3} \frac{m_{2}}{m_{3}}$ and of the same order as the corresponding angle in the original diagonalizing matrix $W$.

In conclusion, when computing the new diagonalization matrix, from the effective mass matrix $H_{e f f}^{d}$, not only do we have to include the extra unitary matrices $K$ and $O_{23}$, but we have also to substitute the new expressions for $\bar{\lambda}$ and $\bar{A}$ by the original $\lambda, A$ in Eq. (7). Thus,

$$
V_{e f f}^{d}=F \bar{W} K O_{23}
$$

where $\bar{W}=W(\bar{\lambda}, \bar{A})$. Obviously, from the form of $V_{e f f}^{d}$, it is clear that CP violation gets an extra contribution as it now includes the complex matrix $K$, which can not be factorized out, i.e. included in redefinitions of the quark fields. It is now easy to see that the leading order term of $I_{C P}$ is just

$$
\begin{aligned}
& \left|I_{C P}\right|=\left(\frac{2 \sqrt{5}}{7} \bar{A}^{2}\right)\left(\frac{2}{\sqrt{5}}\right)\left|\bar{W}_{12}\right|\left(\left|\bar{W}_{12} \bar{W}_{23}\right|+\left|\bar{W}_{13}\right|\right)= \\
& =\left(\frac{2 \sqrt{5}}{7} \bar{A}^{2}\right)\left(\frac{2}{\sqrt{5}}\right) \bar{\lambda}\left(\frac{3}{2} \sqrt{2 A}^{3}\right)= \\
& =\frac{6 \sqrt{2}}{7} \bar{A}^{2} \bar{\lambda}^{6}=\sqrt{\frac{7}{6}}\left(\frac{m_{d}}{m_{s}}\right)\left(\frac{m_{s}}{m_{b}}\right)^{2}
\end{aligned}
$$

where it is sufficient to take the first order terms of $\bar{W}$ in Eq. (8) and substitute the original $\lambda$ and $A$ by the new $\bar{\lambda}, \bar{A}$ in Eq. (23). The factor $\frac{2 \sqrt{5}}{7} \overline{A \lambda}^{2}$ stands for the angle of the extra diagonalization matrix $O_{23}$ and the factor $\frac{2}{\sqrt{5}}$ comes from the contribution from the extra unitary matrix $K$ together with the phases $4 \overline{4}$ of $\bar{W}$. We see now that $I_{C P}$ has become much larger: $\left|I_{C P}\right| \approx o\left(\lambda^{6}\right)$ which is very near to its experimental value $3 \times 10^{-5}$.

\footnotetext{
${ }^{4}$ For the sake of the argument, we have taken until now as an example $W=\mathbb{I}$, however it turns out that, it is the absolute values of the elements of the matrices $W$ that are close to those of the identity. In fact, $W$ itself differs from the identity by a diagonal unitary matrix. To be correct, $W$ is very close to $\operatorname{diag}\left(-i, 1, i e^{i \frac{\pi}{4}}\right)$.
} 


\section{Exact numerical results}

\subsection{Numerical examples}

Next, we present exact numerical results. As previously mentioned, the up quark mass matrix is taken to be a $3 \times 3$ pure USY matrix, given in Eq. (1), while the down quark matrix is a $4 \times 4$ matrix as in Eq. (13). We present 3 kinds of cases and results to be compared:

(i) We give a numerical example with the full $3 \times 4$ CKM matrix and other physical quantities using the full $4 \times 4$ down-type quark mass matrix.

(ii) We calculate the same output using the $3 \times 3$ effective down-type quark mass matrix, according to Eq. (14).

\section{Input:}

$$
\begin{array}{lll}
c_{u}=57.334 \mathrm{GeV} & \alpha_{u}=0.0168 & p_{1}=90+180 i \\
c_{d}=1.163 \mathrm{GeV} & \beta_{u}=0.00088 & p_{2}=-20+125 i \\
& \alpha_{d}=0.108 & p_{3}=-365+20 i \\
& \beta_{d}=-0.028 & \mu_{o}=190
\end{array}
$$

Output (at the $M_{Z}$ scale):

(i) Output using the $4 \times 4$ down-type mass matrix:

$$
\begin{aligned}
& \left(m_{u}, m_{c}, m_{t}\right)=(0.00139,0.610,172) \mathrm{GeV} \\
& \left(m_{d}, m_{s}, m_{b}, m_{D}\right)=(0.00321,0.0529,2.9,553) \mathrm{GeV} \\
& \left|\mathcal{V}_{C K M}\right|=\left(\begin{array}{cccc}
0.97436 & 0.22497 & 0.003606 & 0.0000352 \\
0.22480 & 0.97351 & 0.04163 & 0.000137 \\
0.009371 & 0.04072 & 0.99912 & 0.00350
\end{array}\right), \\
& \rho \equiv\left|\mathcal{V}_{13}\right| /\left|\mathcal{V}_{23}\right|=0.0866, \quad\left|I_{C P}\right|=3.2 \times 10^{-5}, \\
& \sin (2 \beta)=0.685, \quad \gamma=79.2^{\circ} . \\
& \mathrm{FCNC}:\left|\mathcal{U}_{s d}\right|,\left|\mathcal{U}_{d s}\right|=5.3 \times 10^{-10},\left|\mathcal{U}_{b d}\right|,\left|\mathcal{U}_{d b}\right|=8.4 \times 10^{-9},\left|\mathcal{U}_{b s}\right|,\left|\mathcal{U}_{s b}\right|=7.2 \times 10^{-7}
\end{aligned}
$$

(ii) Output using the $3 \times 3$ effective down-type mass matrix approximation of Eq. (15):

$$
\begin{aligned}
& \left(m_{u}, m_{c}, m_{t}\right)=(0.00139,0.610,172) \mathrm{GeV}, \\
& \left(m_{d}, m_{s}, m_{b}\right)=(0.00321,0.0529,2.9) \mathrm{GeV}, \\
& \left|V_{C K M}\right|=\left(\begin{array}{ccc}
0.97436 & 0.22497 & 0.003606 \\
0.22480 & 0.97351 & 0.04163 \\
0.009371 & 0.04072 & 0.99913
\end{array}\right), \\
& \rho \equiv\left|V_{13}\right| /\left|V_{23}\right|=0.0866, \quad\left|I_{C P}\right|=3.2 \times 10^{-5}, \\
& \sin (2 \beta)=0.685, \quad \gamma=79.2^{\circ} .
\end{aligned}
$$


The exact numerical results of Eq. (27) show that a good fit of the quark masses and mixing, including the strength of CP violation can be obtained in the USY framework with the addition of one down-type singlet quark. Comparison of Eqs. (27, 28) shows that the use of the effective down-type mass matrix is an excellent approximation, as anticipated. Furthermore, the off-diagonal elements $\mathcal{U}_{i j}$ of the FCNC mixing matrix are very small. Using the expressions given in [12, and these elements we have computed e.g. the branching ratio of $K^{+} \rightarrow \pi^{+} \nu \bar{\nu}$. We have found that our FCNC mixing matrix elements have no significant influence on the associated EWPM's.

It is important to verify that a good fit of quark masses and mixing, including the strength of $\mathrm{CP}$ violation is obtained in a non-singular region of parameter space, so that the results are stable. This was verified by doing a numerical scan of the parameter space. We chose only a region of the full parameter space where we expect to obtain good results. The input parameter space consists of the parameters $\left(\alpha_{u}, \beta_{u}, \alpha_{d}, \beta_{d}, i p_{1}, i p_{2},-p_{3}, \mu_{o}\right)$. For each of these, we chose initial and final values and a step. We maintained the top and bottom mass at fixed values of 171 and $2.9 \mathrm{GeV}$ 's at $M_{Z}$. We obtained a dense set of points around the values presented in the above example, all of them corresponding to values of masses and mixing allowed by the experimental data.

\section{Conclusions}

We have shown that a good fit of quark masses and mixing, including the strength of $\mathrm{CP}$ violation can be obtained in an extension of the Standard Model with one down-type singlet quark and universal strength of Yukawa couplings connecting standard quarks. The results were obtained both through an analytical approximate diagonalization of the quark mass matrices and through an exact numerical evaluation of quark masses and mixing. The crucial point is that, in the presence of the vector-like quark singlet, the effective $3 \times 3$ quark mass matrix receives in leading approximation two contributions, one arising from the standard quark mass matrices and another one arising from quark mass terms involving the vector-like quark and its mixing with standard quarks. This new contribution to the effective mass matrix is not suppressed by the ratio of the standard quark masses and singlet quark masses. It is this new contribution to the effective quark mass matrix which increases the strength of $\mathrm{CP}$ violation, measured by the rephasing invariant $I_{C P}$. It is worth emphasizing that for a broad class of flavour models, the presence of heavy vectorlike quarks can have an important effect on the structure of low-energy quark masses and mixing. This is an important point, in view of the significant number of flavour models where vector-like quarks are introduced [13].

At this stage the following comment is in order. We have introduced only one isosinglet vectorlike quark. In what concerns the effective quark mixing and $\mathrm{CP}$ violation in the quark sector, this is equivalent to having more than one isosinglet quark, with only one of these new quarks mixing significantly with the standard quarks. Still, we have not imposed the strict USY principle in the mixing terms like $f^{i} \bar{D}_{L} S d_{R_{i}}$ 5. Naively, this could seem unnatural, but this is not the case. Note that for simplicity, we have introduced a $Z_{2}$ symmetry which eliminates some Yukawa couplings, but this is not a crucial ingredient of the model. Without this $Z_{2}$ symmetry, one would have mass terms like $M^{i} \bar{D}_{L} d_{R_{i}}$ which are $S U(2) \times U(1)$ invariant. Consider now that there is a symmetry which would lead to USY in the Yukawa terms connecting the standard quarks. That could be softly broken by the mass terms $\bar{D}_{L} d_{R_{i}}$ without affecting the naturalness of USY in the standard quark sector. On the other hand, if one allows more than one isosinglet quark with significant mixing with standard quarks, one may obtain sufficient $\mathrm{CP}$ violation [11] even with quark mass terms $M^{i} \bar{D}_{L} d_{R_{i}}$ of equal size. The key point of this paper is showing that sufficient $\mathrm{CP}$ violation is generated in the USY framework even if only one isosinglet quark mixes significantly with standard quarks. Such a scenario is entirely plausible if the spectrum of the isosinglet quarks is hierarchical, where one expects that only the lightest isosinglet quark mixes significantly with standard quarks.

It is remarkable that the USY Ansatz implemented in a simple extension of the SM can accommodate the pattern of quark masses and mixing, including the strength of $\mathrm{CP}$ violation. The next step would be to find a framework where the USY structure for the Yukawa couplings would result from a fundamental symmetry, eventually implemented at a higher energy scale. Efforts to derive

\footnotetext{
${ }^{5}$ Even so, it is obvious that imposing the USY structure separately on the $f^{i}$ and $\widetilde{f}^{i}$ of the full interaction term $\bar{D}_{L}\left(f^{i} S+\widetilde{f}^{i} S^{*}\right) d_{R_{i}}$ will result in different absolute values of the respective mass matrix elements.
} 
the USY structure from higher dimension theories have been considered in the literature [14]. It is worth pointing out that, in higher dimensional theories, the Kaluza Klein modes of some fermion fields behave effectively as vectorlike fermions.

\section{Acknowledgements}

This work was partially supported by Fundação para a Ciência e a Tecnologia (FCT, Portugal) through the projects CERN/FP/109305/2009, PTDC/FIS/098188/2008 and CFTP-FCT Unit 777 which are partially funded through POCTI (FEDER) and by Marie Curie Initial Training Network "UNILHC" PITN-GA-2009-237920.

\section{References}

[1] For a recent review and an extended list of references see: G.G. Ross, Journal of Physics: Conference Series 171 (2009) 012006; H. Fritsch, Zhi-zhong Xing, Prog. Part. Nucl. Phys. 45 (2000) 1; A. Strumia and F. Vissani, hep-ph/0606054 K.S. Babu, arxiv.0910.2948; G. Couture et al, Phys. Rev. D 81 (2010) 033010.

[2] G. C. Branco, J. I. Silva-Marcos and M. N. Rebelo, Phys. Lett. B 237 (1990) 446.

[3] Paul M. Fishbane, Peter Kaus, Phys. Rev. D 49 (1994) 4780; J. Kalinowski and M. Olechowski, Phys. Lett. B 251 (1990) 584.

[4] G.C. Branco, J.I. Silva-Marcos, Phys. Lett. B 359 (1995) 166; G.C. Branco, D. EmmanuelCosta, J.I. Silva-Marcos, Phys. Rev. D 56 (1997) 107.

[5] J. I. Silva-Marcos, Phys. Lett. B 443 (1998) 276.

[6] Particle Data Group, J. Phys. G 37, 075021 (2010).

[7] J. A. Aguilar-Saavedra, F.J. Botella and G. C. Branco, Nucl. Phys. B 706 (2005) 204; F.J. Botella, G.C. Branco and M. Nebot Phys.Rev.D79:096009,2009

[8] F. del Aguila and J. Cortes, Phys. Lett. B 156 (1985) 243; G. C. Branco and L. Lavoura, Nucl. Phys. B 278 (1986) 738; F. del Aguila, M. K. Chase and J. Cortes, Nucl. Phys. B 271 (1986) 61; Y. Nir and D. J. Silverman, Phys. Rev. D 42 (1990) 1477; D. Silverman, Phys. Rev. D 45 (1992) 1800; G. C. Branco, T. Morozumi, P. A. Parada and M. N. Rebelo, Phys. Rev. D 48 (1993) 1167; V. D. Barger, M. S. Berger and R. J. N. Phillips, Phys. Rev. D 52 (1995) 1663 arXiv:hep-ph/9503204; M. Gronau and D. London, Phys. Rev. D 55 (1997) 2845 arXiv:hep-ph/9608430; F. del Aguila, J. A. Aguilar-Saavedra and G. C. Branco, Nucl. Phys. B 510 (1998) 39 |arXiv:hep-ph/9703410; G. Barenboim, F. J. Botella, G. C. Branco and O. Vives, Phys. Lett. B 422 (1998) 277 |arXiv:hep-ph/9709369|; G. Barenboim, F. J. Botella and O. Vives, Phys. Rev. D 64 (2001) 015007 |arXiv:hep-ph/0012197|; G. Barenboim, F. J. Botella and O. Vives, Nucl. Phys. B 613 (2001) 285 arXiv:hep-ph/0105306]; K. Higuchi and K. Yamamoto, arXiv:0911.1175 [hep-ph].

[9] F.J. Botella, G.C. Branco, M. Nebot and M.N. Rebelo, Nucl. Phys. B 725 (2005) 155.

[10] G.C. Branco and L. Lavoura, Phys. Lett. B 208 (1988) 123.

[11] K. Higuchi, M. Senami and K. Yamamoto, Phys. Lett. B 638 (2006) 492.

[12] N.G. Deshpande, D.K. Ghosh and X.-G. He, Phys. Rev. D 70 (2004) 093003; J.A. AguilarSaavedra, Phys. Rev. D 67 (2003) 035003;

[13] For recent references see e.g. A.J. Buras, C. Grojean, S. Pokorsky and R. Ziegler, arXiv:1105.3725 [hep-ph]; D. Guadagnoli, R.N. Mohapatra and I. Sung, JHEP 1104 (2011) 093.

[14] P.Q. Hung and M. Seco, Nucl. Phys. B 653 (2003)123; N. Chamoun, S. Khalil, and E. Lashin, Phys. Rev. D 69 (2004) 095011; A. Soddu and N.K. Tran, Phys. Rev. D 69 (2004) 015010. 